\title{
Intestinal Targeting of Ganciclovir Release Employing a Novel HEC-PAA Blended Lyomatrix
}

\author{
Mostafa Mabrouk, ${ }^{1,2}$ Jameel A. S. Mulla, ${ }^{1,3}$ Pradeep Kumar, ${ }^{1}$ Dharmesh R. Chejara, ${ }^{1}$ Ravindra V. Badhe, ${ }^{1}$ \\ Yahya E. Choonara, ${ }^{1}$ Lisa C. du Toit, ${ }^{1}$ and Viness Pillay ${ }^{1,4}$
}

Received 4 September 2015; accepted 19 October 2015; published online 9 November 2015

\begin{abstract}
A hydroxyethylcellulose-poly(acrylic acid) (HEC-PAA) lyomatrix was developed for ganciclovir (GCV) intestine targeting to overcome its undesirable degradation in the stomach. GCV was encapsulated within the HEC-PAA lyomatrix prepared by lyophilization. Conventional tablets were also prepared with identical GCV concentrations in order to compare the GCV release behavior from the lyomatrix and tablets. GCV incorporation $(75.12 \%)$ was confirmed using FTIR, DSC, and TGA. The effect of GCV loading on the microstructure properties of the lyomatrix was evaluated by SEM, AFM, and BET surface area measurements. The in vitro drug release study showed steady and rapid release profiles from the GCV-loaded lyomatrix compared with the tablet formulation at identical $\mathrm{pH}$ values. Minimum GCV release was observed at acidic $\mathrm{pH}(\leq 40 \%)$ and maximum release occurred at intestinal $\mathrm{pH}$ values $(\geq 90 \%)$ proving the intestinal targeting ability of the lyomatrix. Kinetic modeling revealed that the GCV-loaded lyomatrix exhibited zero-order release kinetics $(n=1)$, while the tablets were best described via the Peppas model. Textural analysis highlighted enhanced matrix resilience and rigidity gradient $(12.5 \%, 20 \mathrm{~Pa})$ for the GCV-loaded lyomatrix compared to the pure (7\%, 9.5 Pa) HEC-PAA lyomatrix. Bench-top MRI imaging was used to confirm the mechanism of GCV release behavior by monitoring the swelling and erosion rates. The swelling and erosion rate of the tablets was not sufficient to achieve rapid zero-order GCV release as with the lyomatrix. These combined results suggest that the HEC-PAA lyomatrix may be suitable for GCV intestinal targeting after oral administration.
\end{abstract}

KEY WORDS: ganciclovir; intestine targeting; lyophilized matrix; magnetic resonance imaging; zeroorder release.

\section{INTRODUCTION}

Herpes virus is a popular viral infection of the skin or mucous membranes (1). The lesions produced by herpes virus are overwhelmingly painful, burning, or pruritic, and resort to recur in most patients (2). Short-term therapy with acyclovir can accelerate the healing of an acute outbreak, and continual oral administration of acyclovir is always specified (3). GCV is one of the FDA-approved drugs for treating herpes viruses. However, GCV has poor oral bioavailability (4), owing to its unfavorable degradation in the acidic gastric medium. This necessitates frequent administration of GCV. Recently, increased attention has been provided to polymers that are able

\footnotetext{
${ }^{1}$ Wits Advanced Drug Delivery Platform Research Unit, Department of Pharmacy and Pharmacology, School of Therapeutic Sciences, Faculty of Health Sciences, University of the Witwatersrand, Johannesburg, 7 York Road, Parktown, 2193, South Africa.

${ }^{2}$ Biomaterials Department, National Research Centre, 33 El-Bohouth St. (former El-Tahrir St.), Dokki, Giza, Egypt.

${ }^{3}$ Department of Pharmaceutics, M.M.U. College of Pharmacy, Rajiv Gandhi University of Health Sciences, Bangalore, Karnataka, India.

${ }^{4}$ To whom correspondence should be addressed. (e-mail: viness.pillay@wits.ac.za)
}

to alter their volume and properties in response to $\mathrm{pH}$ stimulation for application in the design of specialized gastroprotective oral drug delivery systems (5-8).

Polyacrylic acid (PAA) possesses many desirable properties including the ability to exist as a liquid at $\mathrm{pH} \leq 5$ and as a gel at $\mathrm{pH} \geq 7$ (9). The permeation of cations into the gelled polymer converts the gel back to a liquid (10). Another polymer, hydroxyethylcellulose (HEC), is one of the most widely used cellulose derivatives with wide application in pharmaceutical formulation design, due to its non-toxicity and relatively low cost. As sensitivity to $\mathrm{pH}$ is required for optimal intestinal drug targeting, many polymers have been investigated (1013). Such polymers demonstrated optimal swelling behavior at neutral $\mathrm{pH}$ with low swelling ratios at acid $\mathrm{pH}$ (9). Furthermore, the water sorption potency of the polymers can be adjusted and enhanced via introducing interconnecting pores (14-16).

The development of a specialized oral drug delivery formulation for zero-order release of GCV can decrease the frequency of dosing in a manner that is safe and non-toxic. Zero-order release is the most favorable kinetic pattern of drug release in order for drugs to overcome Fick's law. Two common techniques have been used to design zero-order drug release formulations. One involves combining two different 
surfaces through which drug must traverse to reach a holding volume and then move out to the delivery site (17). The second technique is via preparation of a system through which drug is released by means of more than one mechanism. The incorporation of two mechanisms can, under well-defined conditions, provide a release profile which is relatively constant. Biphasic polymeric matrices have exploited the second technique as previously reported (18).

Recently, improving the properties of conventional polymer matrices, especially the response rate, has been explored through several techniques such as the formation of porous/super-porous structures (19), the addition of polymer particles within the polymer network, as well as cold treatment (20) or freeze-drying of the swollen polymer matrix (21). The physically crosslinked polymer matrices can maintain their physical immutability due to the existence of reversible physical junction fields related to hydrogen bonding, hydrophobic interactions, chain entanglements, crystallinity, and/or ionic complication (22). However, either PAA or HEC alone cannot support zero-order site-specific release of GCV to the small intestine. Therefore, the development of a blended HEC-PAA lyophilized matrix (termed a lyomatrix from here on) may provide desirable zero-order GCV release as well as intestinal targeting due polymeric property synergism.

The present study is the first report on the use of a HECPAA lyomatrix blend as a platform for site-specific zero-order release of GCV targeted to the small intestine after oral administration. The lyomatrix was prepared by blending and lyophilization of PAA and HEC with the aim of exploiting and enhancing the properties of both polymers as a block copolymeric structure. Polyvinyl alcohol (PVA) was utilized as a plasticizer and a physical crosslinker due to its hydrogen bonding capability. The lyomatrix was loaded with GCV before the lyophilization process. GCV incorporation into the lyomatrix was investigated using Fourier transform infrared spectroscopy (FTIR), differential scanning calorimetry (DSC), and thermogravimetric analysis (TGA). The effect of GCV addition on the microstructure and lyomatrix resilience was evaluated qualitatively and quantitatively by scanning electron microscope (SEM), atomic force microscope (AFM), Brunauer-Emmett-Teller (BET) surface area measurements, and textural analysis. The lyomatrix formed was also evaluated to determine the in vitro drug release profile. Herein, it was also hypothesized that the GCV release behavior, the lyomatrix swelling behavior, and the erosion rate were highly dependent on the formulation type (the lyomatrix $v s$. a conventional tablet system). Therefore, equivalent formulations were prepared in tablet form with the same GCV concentrations for comparison with the newly synthesized lyomatrix. The tablets were then also subjected to SEM, in vitro drug release analysis, and monitoring of hydration and erosion kinetics via bench-top magnetic resonance imaging (MRI) in order to prove this hypothesis.

\section{MATERIALS AND METHODS}

\section{Materials}

Polyacrylic acid (PAA) (average $\mathrm{M}_{\mathrm{w}}=450,000 \mathrm{~g} / \mathrm{mol}$ ) and 2-hydroxyethylcellulose $\left(M_{w}=90,000 \mathrm{~g} / \mathrm{mol}\right)$ were purchased from Sigma-Aldrich (St. Louise, MO, USA). Polyvinyl alcohol (PVA) (87-89\% hydrolyzed) (average $\mathrm{M}_{\mathrm{w}}=13,000-23,000 \mathrm{~g} / \mathrm{mol}$ ) was also purchased from Sigma-Aldrich (St. Louise, MO, USA). Ganciclovir (GCV) $500 \mathrm{mg}$ intravenous infusion was purchased from Roche Products (Pty) Ltd/(Edms) Bpk (Australia). The water used was Milli-Q water from a Millipore Water Purification System.

\section{Synthesis of the HEC-PAA lyomatrix and GCV-loaded formulations}

The lyomatrix was prepared as follows: PAA and HEC (1:1) were dissolved in $50 \mathrm{~mL}$ of Milli-Q water to prepare a $10 \%$ blend at $70^{\circ} \mathrm{C}$ until a homogenous solution was obtained. Thereafter, $10 \mathrm{~mL}$ of PVA $(10 \%)$ solution was added to the HEC/PAA polymer blend and stirred until the viscosity increased at room temperature $\left(21^{\circ} \mathrm{C}\right)$. GCV was then loaded in the resulting solution, and the drug to polymer ratios were kept at 1:10 and 1:5. The resultant mixture was then frozen for $24 \mathrm{~h}$ at $-80^{\circ} \mathrm{C}$ and thereafter lyophilized (Virtis2KBTXL-75 Benchtop SLC Freeze Dryer, UK) for $24 \mathrm{~h}$ at $-64^{\circ} \mathrm{C}$. Identical formulations were also prepared by incorporation of GCV after the lyophilization process followed by conventional tablet preparation. All samples were kept under a desiccator until further characterization. GCV-loaded formulations prepared are listed in Table I.

\section{Determination of GCV-Loading Efficiency within the HEC- PAA Lyomatrix}

The quantity of GCV loaded in the HEC-PAA lyomatrix was ascertained by grounding GCV-loaded samples and extraction of GCV in methanol by gentle heat over $12 \mathrm{~h}$. The solution was then filtered, diluted with PBS, and analyzed by UV spectrophotometry (Lambda $25 \mathrm{UV} / \mathrm{Vis}$ Spectrophotometer, PerkinElmer, MA, USA) at a $\lambda_{\max }$ of $254 \mathrm{~nm}$ using a calibration curve. All measurements were conducted in triplicate, and the $\%$ GCV-loading and encapsulation efficiency were calculated using Eqs. 1 and 2.

$\% \mathrm{GCV}$-loading $\frac{\text { Weight of GCV in formulation }}{\text { Weight of total formulation }} \times 100$

$\%$ Encapsulation Efficiency $\frac{\text { Actual GCV loading }}{\text { Theoretical GCV loading }} \times 100(2)$

Table I. List of Various GCV-Loaded Formulations Prepared

\begin{tabular}{lll}
\hline Sample & Lyomatrix (\%) & $\begin{array}{c}\text { Ganciclovir } \\
\text { loading (\%) }\end{array}$ \\
\hline Drug-free HEC-PAA lyomatrix & 100 & - \\
Lyomatrix formulation 1 (LMF1) & 90 & 10 \\
Lyomatrix formulation 2 (LMF2) & 80 & 20 \\
Tablet formulation 1 (TF1) & 90 & 10 \\
Tablet formulation 2 (TF2) & 80 & 20 \\
\hline
\end{tabular}


Assessment of the Chemical Integrity of the GCV-Loaded HEC-PAA Lyomatrix

In order to assess the effect of the lyomatrix on GCV stability and integrity, ATR-FTIR spectra were recorded for the lyomatrix formulations (LMF1 and LMF2) for characteristic functional group determination using a Perkin Elmer Spectrum 2000 FTIR spectrometer, employing a singlereflection diamond MIRTGS detector (PerkinElmer Spectrum 100, Lantrisant, Wales, UK). All samples were analyzed by a universal ATR polarization accessory for the FTIR spectrum series at a resolution of $4 \mathrm{~cm}^{-1}$. Specimens were placed on a diamond crystal running each sample at 100 iterations to reduce the signal to noise ratio to a minimum of 10 , in the range of $4000-600 \mathrm{~cm}^{-1}$ under a constant pressure of 120 psi.

\section{Thermal Characterization of the GCV-Loaded HEC-PAA Lyomatrix}

In order to examine the influence of the lyomatrix on the GCV crystallinity, the thermal behavior of the lyomatrix before and after GCV loading was determined by differential scanning calorimetry (Mettler Toledo, DSC1, STARe System, Schwerzenback, Switzerland). Samples $(10 \pm 1 \mathrm{mg})$ were placed into $40-\mu \mathrm{L}$ aluminum pans and heated from 20 to $500^{\circ} \mathrm{C}$ at a heating rate of $10^{\circ} \mathrm{C} / \mathrm{min}$. In addition, TGA of samples was undertaken to evaluate the lyomatrix formulation stability as a function of temperature under a $\mathrm{N}_{2}$ atmosphere with a flow rate of $30 \mathrm{~mL} / \mathrm{min}$ and heating rate of $10^{\circ} \mathrm{C} / \mathrm{min}$. Pyris6 software was used to perform the thermal analysis. Heating was ramped from 30 to $900^{\circ} \mathrm{C}$. All tests were undertaken in triplicate $(N=3)$.

\section{Evaluation of GCV Loading on the Morphology and} Microstructure of the HEC-PAA Lyomatrix

In order to determine the effect of GCV loading on the morphology and microstructural properties of the lyomatrix formulations, the microarchitecture before and after GCV loading was qualitatively assessed by SEM and AFM. Quantitative analysis was performed by determining the surface area, average pore diameter, and total pore volume employing BET surface area measurements. The lyomatrix formulations before and after GCV loading were investigated using a Phenom ${ }^{\mathrm{TM}}$ SEM (FEI Company, OR, USA). The tablet formulations (TF1 and TF2) were also visualized for comparison with the internal structure of the lyomatrix formulations (LMF1 and LMF2). The lyomatrix formulations were coated with gold using a gold-sputter coating (SPI Module $^{\text {TM }}$ Sputter Coater, SPI Supplies, PA, USA) and were fixed on the SEM sample holders using adhesive carbon tape. In addition, the surface topography transitions of the lyomatrix before and after GCV loading were observed by AFM with a tapping mode in air using a Dimension 3100 Veeco AFM. All images were generated using commercial high-resolution tapping mode silicon probes with an 8-nm tip radius. The free air oscillation amplitude was typically $75 \%$, and the cantilever resonance frequency was $300 \mathrm{kHz}$. The BET surface area measurements were measured using a Porositometric Analyzer (Micromeritics ASAP2020,
Norcross, GA, USA), whereby $100 \mathrm{mg}$ of samples underwent two stages of analysis, firstly, following a degassing phase and thereafter an absorption and desorption cumulative phase for accurate analysis of the surface area and micrometric parameters.

\section{Comparative In Vitro GCV Release Analysis from HEC- PAA Lyomatrix and Tablet Formulations}

GCV release studies were undertaken using a USP 33 apparatus II (Erweka DT 700 GmbH Germany). GCV-loaded formulations $(N=3)$ were submerged in the dissolution vessel and covered with a stainless steel ring-mesh assembly in order to avoid the paddle inflicting physical $/$ mechanical damage to the samples and potentially influencing the release profiles obtained due to disturbed hydrodynamics (23). All vessels were comprised $900 \mathrm{~mL}$ simulated gastrointestinal fluid (GIF) at varying $\mathrm{pH}$ values $(\mathrm{pH} 1.2,4,6.8$, and 7.4$)$ at $37^{\circ} \mathrm{C}$. The rotating paddle was adjusted at $50 \mathrm{rpm}$, and the dissolution apparatus was calibrated for a $4 \mathrm{~h}$ run at $\mathrm{pH} 1.2,4 \mathrm{~h}$ at $\mathrm{pH} 4,8 \mathrm{~h}$ at $\mathrm{pH} 6.8$, and $8 \mathrm{~h}$ at $\mathrm{pH} 7.4$ with samples taken at $1-\mathrm{h}$ intervals. Sampling involved the removal of $5 \mathrm{~mL}$ of dissolution media with subsequent replacement of fresh buffer in order to preserve sink conditions. Samples were then measured by UV spectroscopy to determine the GCV concentration at $254 \mathrm{~nm}$ as previously reported (24). In order to compare the release behavior of the GCV-loaded lyomatrix formulations (LMF1 and LMF2) with the tablet formulations (TF1 and TF2), the corresponding tablet formulations containing the same GCV concentrations as the lyomatrix formulations were prepared for drug release analysis.

\section{Analysis of the GCV Release Kinetics from the HEC-PAA Lyomatrix}

The release data of the lyomatrix formulations were modeled using different kinetic equations with a view to determine their mechanisms and kinetics of drug release. In addition, GCV release from unsophisticated systems may be described by the famous power law Eq. 3 (25):

$M_{\mathrm{t}} / M_{\infty}=K_{1} t^{n}$

where $M_{\mathrm{t}}$ and $M_{\infty}$ are the quantities of the released drug at time $t$ and the overall quantity released, respectively; $K_{1}$ is a release constant; and $n$ is a release exponent indicative of the release mechanism. In order to predict the diffusion behavior, the Peppas-Sahlin Eq. 4 may be used (26).

$M_{\mathrm{t}} / M_{\infty}=K_{1} t^{n}+K_{2}^{2 n}$

where $K_{1}$ is the Fickian kinetic constant and $K_{2}$ is the relaxational/dissolution rate constant (i.e., anomalous transport). Drug release from devices with surface erosion and different geometries also have been analyzed by the Hopfenberg Model (27) that proposes applicability to either a slab, cylinder, or sphere showing heterogeneous erosion (Eq. 5).

$M_{\mathrm{t}} / M_{\infty}=1-\left(1-K_{1} t\right)^{n}$ 
where $K_{1}$ is the erosion rate constant. Moreover, appropriate model diagnostic requires the use of diagnostics such as the correlation coefficient, sum of squares of residuals (SS), standard error of weighted residuals (SE), Akaike Information Criterion (AIC), and Schwarz Bayesian Criterion (SBC). AIC and SBC values hold the most significance (28). WinNonlin Linear Mixed Effects module (WinNonlin ${ }^{\circledR}$ V5.3, Pharsight Software, Apex, NC, USA) was used and instituted the smaller-is-better form of the AIC shown in Eq. 6.

$$
\mathrm{AIC}=2 L_{R}+2 s
$$

where $L_{R}$ is the restricted log-likelihood function evaluated at final fixed parameter estimates and the final variance parameter estimates, and ' $s$ ' is the rank of the fixed effects design matrix $X$ plus the number of parameters in $\theta$ (i.e., $s=$ $\operatorname{rank}(X)+\operatorname{dim}(\theta))$.

The linear mixed effects module also uses the smaller-isbetter form of the SBC shown in Eq. 7.

$$
\mathrm{SBC}=-2 L_{R}+\operatorname{slog}(N-r)
$$

where $L_{R}$ is as described above, $N$ is the number of observations used, $r$ is the rank of the fixed effects design matrix $X$, and $s$ is the rank of the fixed effects design matrix $X$ plus the number of parameters $\theta$ (i.e., $s=\operatorname{rank}(X)+\operatorname{dim}(\theta)$ ).

\section{Analysis of the Physicomechanical Behavior of the HEC- PAA Lyomatrix}

Matrix resilience (MR) could be defined as the competence of the lyomatrix to realize energy while elastically deformed and release that energy after unloading. The rigidity gradient (RG) refers specifically to unit migration guided by gradients in lyomatrix rigidity. The deformation energy (DE) is defined as the absorbed energy that causes deformation of the lyomatrix (29). In order to determine the influence of GCV loading on the physicomechanical properties of the lyomatrix formulations, the $\mathrm{MR}, \mathrm{RG}$, and DE values were determined from textural profiles generated before and after GCV loading using a Texture Analyser (TA.XTplus, Stable Microsystems, Surrey, UK). A compressive stress was applied by a textural probe onto the lyomatrix samples with dimensions of $(10 \times 10 \mathrm{~mm})(\mathrm{L} \times \mathrm{D}) \mathrm{ac}-$ cording to the following conditions: pre-test speed $=0.5 \mathrm{~mm} / \mathrm{s}$, starting compression force $=1 \mathrm{~kg}$, distance $=5 \mathrm{~mm}$ ( $50 \%$ of sample length), test speed $=1 \mathrm{~mm} / \mathrm{s}$, and acquisition rate $=200$ points s. Thereafter, the samples were allowed to recover to their original dimensions. The MR (\%) was assessed according to the GCV concentration at $25^{\circ} \mathrm{C}$.

\section{MRI of the Hydrodynamic Behavior of the GCV-Loaded Tablet Formulations}

As indicated, the reason for comparing the lyomatrix formulations (LMF1 and LMF2) with the tablet formulations (TF1 and TF2) was to prove the hypothesis that the swelling and erosion dynamics were highly dependent on the formulation type (lyomatrix or tablet) and significantly contributed to mechanism of achieving zero-order GCV release from the HEC-PAA lyomatrix. To evaluate the hydrational swelling and erosion rate, tablets with dimensions of $(1 \times 5 \mathrm{~mm})(\mathrm{L} \times$ D) were prepared from the lyomatrix and were loaded with identical GCV concentrations. A digital MARAN-ip System configured with a DRX2 HF spectrometer console (Oxford Instruments Magnetic Resonance, Oxon, UK) and with a compact 0.5 Tesla permanent magnet stabilized at $37^{\circ} \mathrm{C}$ was used to generate in situ images of the swelling and erosion dynamics of the tablet formulations. Monitoring of the swelling and erosion behavior of the GCV-loaded tablets was undertaken in $\mathrm{PBS}\left(\mathrm{pH} 6.8 ; 37^{\circ} \mathrm{C}\right)$. The cone-like lower region of the cell was filled with glass beads to provide laminar flow at $16 \mathrm{~mL} /$ min of the used solvents. This was undertaken after configuring, optimizing the shims, and probe tuning. The tablet formulations were then placed in position each time within the cell which in turn was positioned in a magnetic bore, and magnetic resonance images were acquired every 10 min over $24 \mathrm{~h}$ with MARAN-iV1.0 software. The images were obtained after setting the frequency offset and testing gain utilizing RINMR V5.7 under a continuous solvent flow with bio-relevant buffers.

\section{RESULTS AND DISCUSSION}

\section{Assessment of the Synthesized HEC-PAA Lyomatrix}

The method used for preparation of the HEC-PAA lyomatrix loaded with GCV did not include the use of any organic solvents. Moreover, the entrapment efficiency values ranged from 73.41 to $75.12 \%$. It is worth highlighting that the GCV concentration and lyophilization technique had no effect on the entrapment efficiency. Lyophilization has an advantage in that the GCV was exposed to lower thermal stress. A further advantage is that the risk of phase segregation was reduced directly as the solution was vitrified (29).

\section{Evaluation of the Vibrational Transitions of the GCV-Loaded Lyomatrix}

FTIR bands of the lyomatrix, pure GCV, and the GCVloaded lyomatrix are presented in Table II. The transmission spectra of the lyomatrix before and after drug loading are shown in Fig. 1. Both F1 (10\% GCV) and F2 (20\% GCV) exhibited typically identical bands for the native HEC-PAA lyomatrix, suggesting that GCV was encapsulated inside the lyomatrix without any observed effect on the chemical integrity of GCV.

\section{Thermal Analysis of the GCV-Loaded HEC-PAA Lyomatrix}

It is well known that the crystallinity of drug is an important factor in drug solubility. Therefore, the thermal behavior of the GCV-loaded lyomatrix was determined using DSC analysis to investigate the lyomatrix effect on the crystallinity of loaded GCV. An endothermic peak was observed at approximately $100^{\circ} \mathrm{C}$ for all samples due to the minute quantity of moisture present in the samples as shown in Fig. 2a. Pure GCV was amorphous with a $T_{\mathrm{g}}$ of $50 \pm 4^{\circ} \mathrm{C}$, and a broad melting endothermic peak between 270 and $315^{\circ} \mathrm{C}$ was recorded as previously reported $(4,34)$. The HEC-PAA lyomatrix and GCV-loaded lyomatrix showed identical crystallization behavior. It was notable that the increase in the melting endotherm of the GCV-loaded samples $\left(320^{\circ} \mathrm{C}\right)$ compared to both pure $\mathrm{GCV}\left(254^{\circ} \mathrm{C}\right)$ and the HEC-PAA lyomatrix $\left(230^{\circ} \mathrm{C}\right)$ 
Table II. FTIR Band Designations for the HEC-PAA Lyomatrix Before and after GCV Loading

\begin{tabular}{llll}
\hline Material & Wavenumber $\left(\mathrm{cm}^{-1}\right)$ & Group assignment & References \\
\hline HEC-PAA, HEC & 1355 & O-H bending & $(30)$ \\
HEC-PAA, HEC & 2929 & Aliphatic C-H stretching & $(30)$ \\
HEC-PAA, HEC & 1429 & C-H bending vibrations & $(30)$ \\
HEC-PAA, HEC & 1020 & C-O-C stretching & $(30)$ \\
HEC-PAA, HEC & 1050 & C-O-C vibrations & $(30)$ \\
HEC-PAA, HEC & 1145 & C-O stretching vibrations & $(30)$ \\
HEC-PAA, HEC & 2943 & stretching of aliphatic $-\mathrm{CH}_{2}-, \mathrm{C}^{-\mathrm{H}}$ & $(30)$ \\
HEC-PAA, HEC & 1257 & Ethyl groups & $(30)$ \\
HEC-PAA, PAA, PVA & 1720 & C=O vibration stretching & $(31,32)$ \\
HEC-PAA, PAA & 3065 & O-H stretching & $(31)$ \\
HEC-PAA,PAA & 1410 & COO- asymmetrical \\
HEC-PAA,PAA & 1472 & COO- symmetrical \\
HEC-PAA,PVA & 820 & "OH" & $(31)$ \\
HEC-PAA,PVA & 3470 & Stretching vibration of the OH \\
GCV & $3333 \mathrm{~cm}$ & NH stretch vibration & $(32)$ \\
GCV & 1580 & $\mathrm{C}=\mathrm{O}$ stretching vibration \\
GCV & 1073 and 1037 & CH deformation vibrations & $(32)$ \\
GCV & 3540 and 2990 & O-H stretching vibration broad band \\
HEC-PAA,GCV & 1570 & C=O stretching vibration & $(33)$ \\
HEC-PAA,GCV & 970 and 1150 & C-O stretching vibrations & $(33)$ \\
\hline
\end{tabular}

suggested an elevation of crystallinity. The thermal transition of the GCV may be attributed to the increased interaction between modular groups of the lyomatrix with pure GCV which hindered the free rotation of the polymeric chains in the lyomatrix. Moreover, TGA results revealed that the initial mass loss of 2$5 \%$ was due to physisorbed water, which was followed by mass loss due to $\mathrm{GCV}>100-300^{\circ} \mathrm{C}$. The lyomatrix decomposed above $300^{\circ} \mathrm{C}$ and completely degraded at $450^{\circ} \mathrm{C}$ as shown in Fig. 2 b. A similar result was reported by Ganesh and co-workers (35).

\section{Morphological Assessment of the GCV-Loaded Lyomatrix}

Figure 3 depicts the SEM images of the lyomatrix, pure GCV, and GCV-loaded samples. The SEM image of pure GCV exhibited small fragment-like particles (Fig. 3a). The HEC-PAA lyomatrix

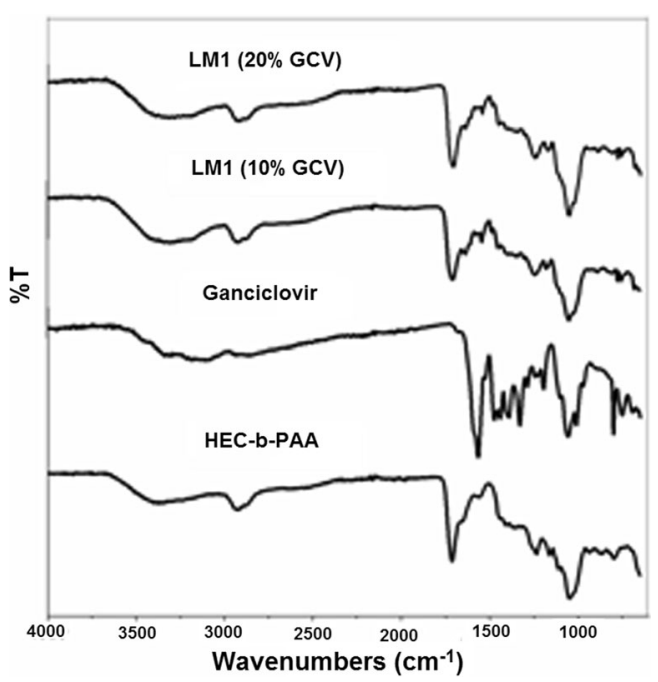

Fig. 1. FTIR spectra of the prepared HEC-PAA lyomatrix before and after GCV loading showed a highly interconnected porous structure (Fig. 3b). The pores were the regions of water permeation and interaction sites for external stimuli with hydrophilic groups of the lyomatrix (31). The GCV-loaded lyomatrix demonstrated differing morphology compared with the native lyomatrix (Fig. 3e) due to the presence of GCV. As expected, a compact morphology was observed for the tablets loaded with GCV (Fig. 3d). The different morphology and microstructure for both the lyomatrix and tablet explains and supports the hypothesis that swelling, erosion rate, and release behavior were affected by the formulation type. In other words, the porous structure of the newly formed lyomatrix provided an advantage over the conventional tablet formulation, as the tablet required more time to form channels for GCV release, whereas the lyomatrix architecture enabled immediate GCV release.

To obtain additional evidence on GCV incorporation into the lyomatrix, AFM spectroscopy was undertaken. From a viewpoint of direct observation of polymeric systems, a lyomatrix can be relatively easily identifiable via such observations since they have a characteristic microstructure partitioned by the constituent polymer chains. Several studies have been reported using AFM aimed to directly observe the characteristic mesh structure of polymer matrices (36-39). SEM images of the lyomatrix before and after GCV loading exhibited a porous and rough morphology compared with the traditional tablet. Therefore, AFM analysis was conducted. AFM offered the unique possibility to quantifying the roughness of the surfaces with high resolution, thus allowing evaluation of the changes promoted by GCV incorporation. The HEC-PAA lyomatrix exhibited high roughness owing to its large porous structure as confirmed by SEM images. Upon loading of GCV, an important alteration in surface topography was achieved. A higher number of smaller grains agglomerated into chain-like structures producing a smoothed GCV-loaded lyomatrix surface, as illustrated in Fig. 3c, f. Similar to these findings, Milosavljevi and co-workers (40) explained the presence of small grain aggregates on the surface of a hydrogel due to $\mathrm{Cd}^{2+}$ ions adsorption. In this case, the presence of grains and the flattened surface is due to GCV entrapment. 
a

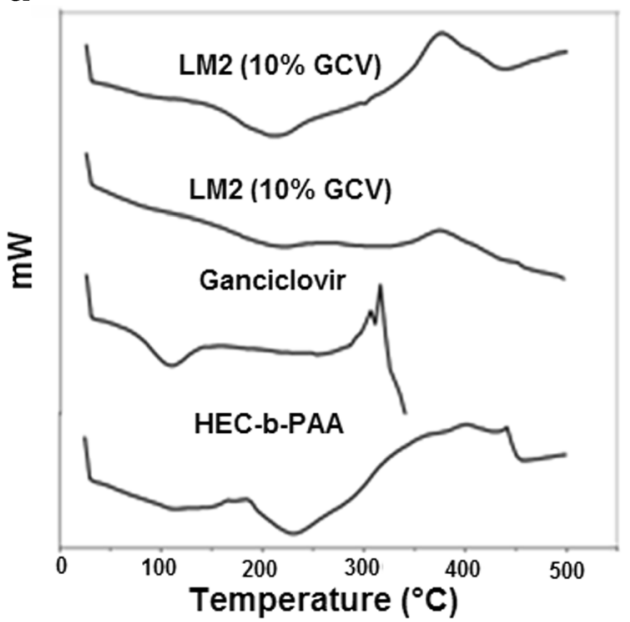

b

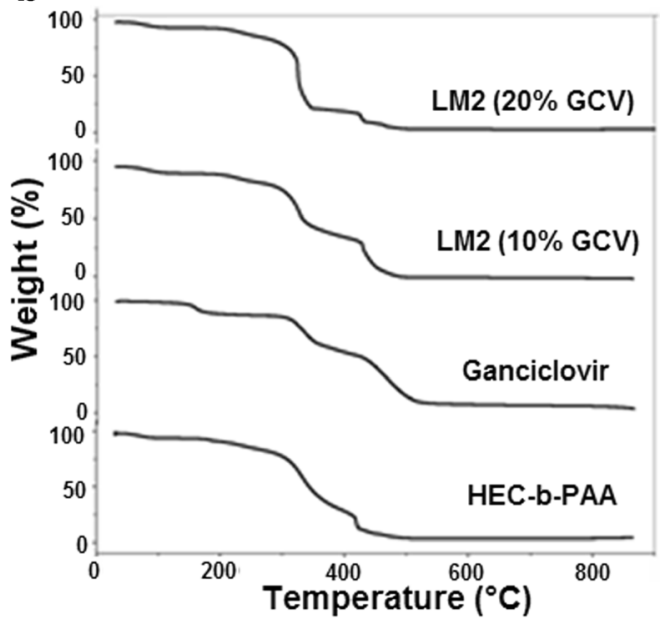

Fig. 2. DSC thermograms of a HEC-PAA lyomatrix before and after GCV-loading and b TGA of HECPAA lyomatrix before and after GCV loading

BET Surface Area Measurements of the GCV-Loaded Lyomatrix

The effect of GCV loading on the lyomatrix was quantitatively estimated using BET surface area measurements. Table III demonstrates the effect of GCV incorporation on the BET surface area, average pore diameter, and total pore volume. It is worth noting that the higher GCV concentration the lower surface area and porosity. As was expected, the tablets exhibited very low surface area and porosity compared with the lyomatrix. These results are consistent with the SEM and AFM
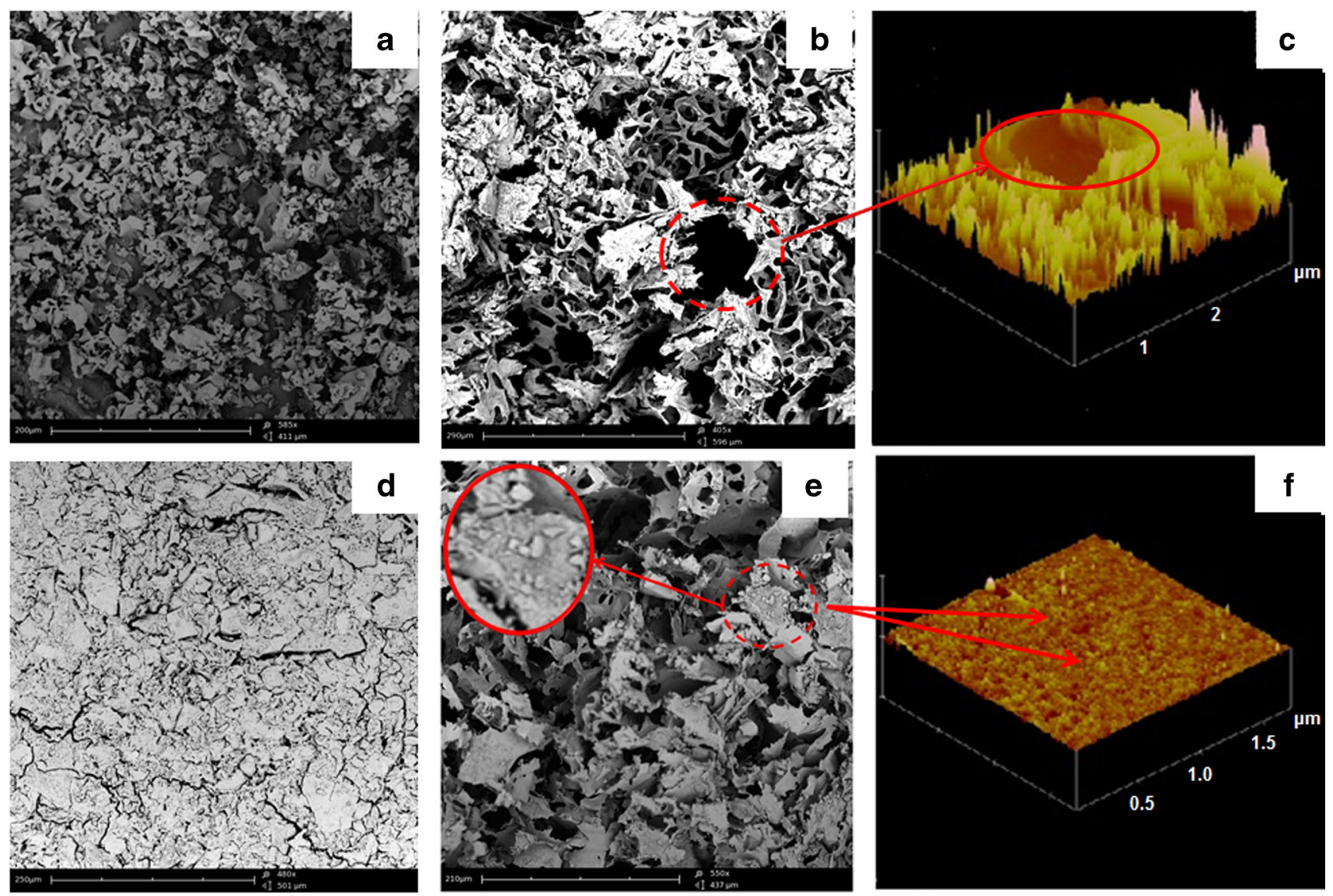

Fig. 3. Morphological characterizations of the lyomatrix: a SEM image of pure GCV, b SEM image of HEC-PAA lyomatrix, c AFM image of HEC-PAA lyomatrix, d SEM image of GCV-loaded tablet, e SEM image of GCV-loaded HEC-PAA lyomatrix, and $\mathbf{f}$ AFM image of GCVloaded HEC-PAA lyomatrix 
Table III. BET Surface Area and Porosity Measurements of the HEC-PAA Pre- and Post-GCV Loading

\begin{tabular}{lccc}
\hline Sample & BET surface area $\left(\mathrm{m}^{2} / \mathrm{g}\right)$ & Average pore diameter $(\AA)$ & Total pore volume $(\AA)$ \\
\hline HEC-PAA lyomatrix & $4.782 \pm 0.197$ & 125.661 & 8522.666 \\
Lyomatrix formulation 1 (LMF1) & $4.448 \pm 0.195$ & 80.546 & 8150.423 \\
Lyomatrix formulation 2 (LMF2) & $4.214 \pm 0.201$ & 74.048 & 8080.956 \\
Tablet formulation 1 (TF1) & $0.690 \pm 0.004$ & 29.046 & 469.547 \\
Tablet formulation 2 (TF2) & $0.606 \pm 0.036$ & 20.358 & 432.153 \\
\hline
\end{tabular}

results. The microarchitecture properties of the GCVloaded samples were highly affected by the presence of GCV. The matrix resilience, swelling, and erosion were also affected by the GCV presence, which in turn altered the drug release behavior as reported previously (14-16).

\section{In Vitro Drug Release Studies}

Comparative drug release studies were performed on the GCV-loaded lyomatrix and tablet formulation. Cumulative drug release as a function of time is shown in Fig. 4. All
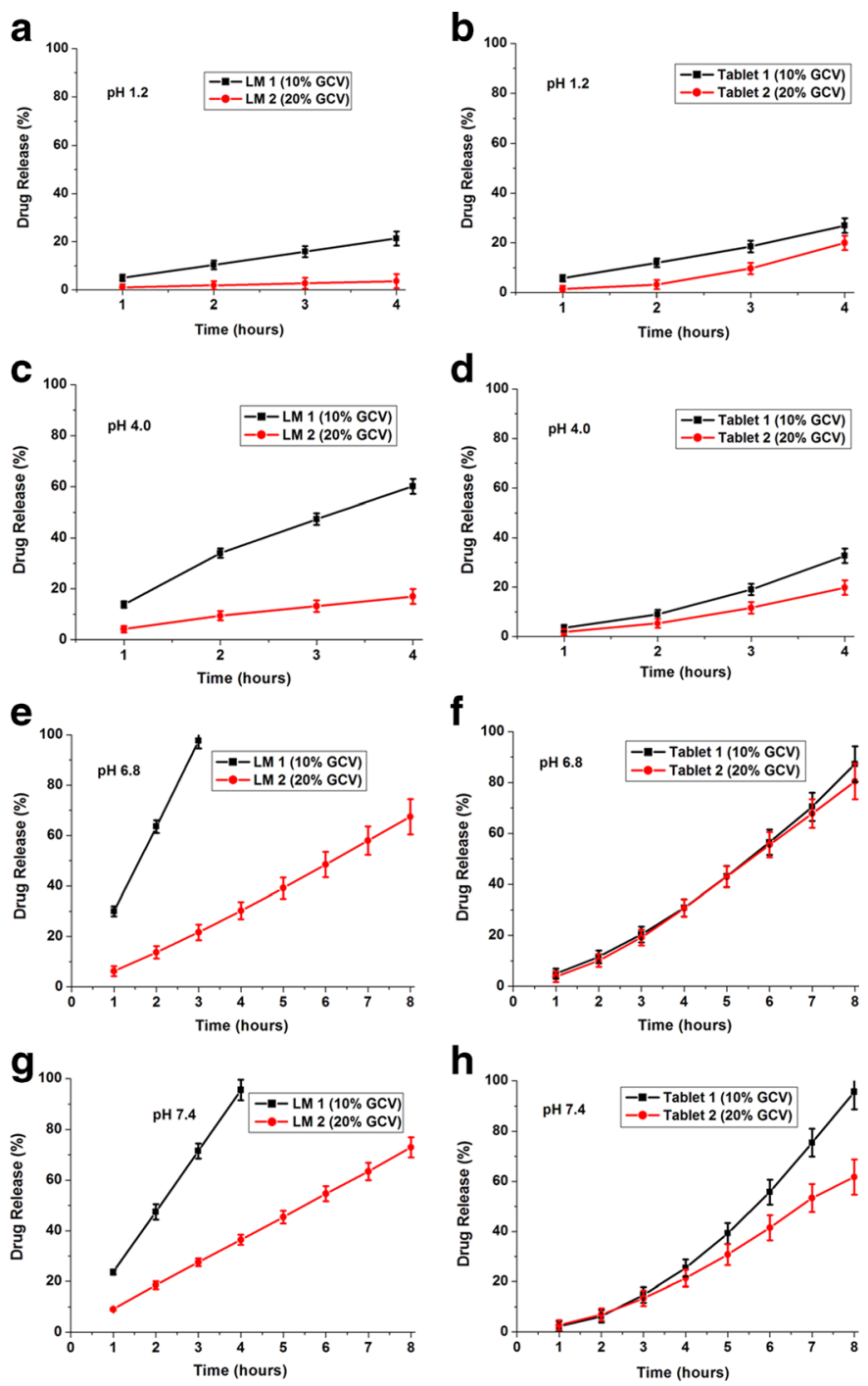

Fig. 4. Cumulative drug release profiles of the prepared samples at varying $\mathrm{pH}$ values a lyomatrix ( $\mathrm{pH}$ 1.2), b tablet ( $\mathrm{pH}$ 1.2), c lyomatrix $(\mathrm{pH} 4.0)$, d tablet at $\mathrm{pH}(4.0)$, e lyomatrix ( $\mathrm{pH} 6.8), \mathbf{f}$ tablet ( $\mathrm{pH} 6.8), \mathbf{g}$ lyomatrix ( $\mathrm{pH} 7.4)$, and $\mathbf{h}$ tablet $(\mathrm{pH} 7.4)$ 
samples exhibited minimum release of $20-60 \%$ at acidic $\mathrm{pH}$ (1.2 and 4.0) as shown in Fig. 4a, b, c, and d. On the other hand, all samples showed maximum release at neutral and slightly alkaline $\mathrm{pH}$ (6.8 and 7.4) as shown in Fig. 4c, d confirming the intestinal targeting of GCV from the lyomatrix system. Generally, all GCV-loaded lyomatrix samples displayed rapid zero-order release over $4 \mathrm{~h}$ up to $100 \%$. However, the tablet formulation showed controllable up curving release characteristics with $90 \%$ of GCV released over 8 h. These results could be explained owing to the highly porous nature of the lyomatrix compared to the compact nature of the traditional tablets (Fig. 3d) and as reported previously (3642). It is also highlighted that PAA is a highly super absorbent polymer. In addition, changing the $\mathrm{pH}$ of the medium significantly affected the swelling capacity. The lyomatrix incorporates carboxylate groups within its structure due to the presence of PAA. The acidic $\mathrm{pH}$ environment prevented ideal anion-anion repulsion of the carboxylate groups. The shielding of the carboxylate groups decreased the swelling capacity. Furthermore, it had been reported previously that at acidic $\mathrm{pH}$ values, low electrostatic repulsion occurs between the carboxylic acid groups of the PAA backbone, consequently decreasing polymeric matrix swelling and minimizing drug release by diffusion (42). Conversely, in alkaline media, the $\mathrm{OH}$-presence elevates the electrostatic repulsion between carboxylate groups, raises the swelling ability, and thus increasing drug release (43). The drug release studies at different $\mathrm{pH}$ values indicated that lower release was observed at gastric $\mathrm{pH}$ with maximum release at intestinal $\mathrm{pH}$ values (4.0-6.8). These in vitro results may be used further as a prediction for GCV in vivo drug release behavior and bioavailability enhancement of GCV after oral dosing.

\section{Analysis of Drug Release Kinetics}

Tables IV and $\mathrm{V}$ list the values from applying the release data to different kinetic models. The diffusion exponent of the GCV-loaded tablet formulation was found to be $>1(n>1)$ (Table V) with comparatively low AIC, SBC, and higher $R^{2}$ values. These results indicated super case II transport (Korsmeyer-Peppas model and or Peppas-Sahlin model) which revealed that the tablet release behavior was due to a combination of diffusion into the swollen polymer and the polymer relaxation process (26-28). In super case II transport, the release curve was linear for an exponential function of the release versus time. The slower release rate of GCV from the tablets, compared with the lyomatrix, was due to more time consumed in creating pores and channels and consequently supporting solvent permeation with slow increments in drug release. On the other hand, the diffusion exponent (Table IV) was approximately equal to $1(n=1)$ for the GCV-loaded lyomatrix with comparatively low AIC, SBC, and higher $R^{2}$ values. This implied that the release of GCV was case II or zeroorder transport. For systems exhibiting case II transport, the dominant mechanism for drug transport is due to polymer matrix relaxation as reported previously $(9,35)$. The higher and more constant release observed for the GCVloaded lyomatrix was due to the interconnected highly porous structure compared to the tablet formulation as confirmed by the SEM and AFM results. These pores facilitated the rapid hydration and swelling of the lyomatrix which in turn enhanced the release behavior of GCV from the lyomatrix. To date, the available excipients for oral drug delivery of GCV do not fully assure the advantages of zero-order GCV release with low release at gastric $\mathrm{pH}$ (1.2) compared with intestinal $\mathrm{pH}$ values (4.06.8). Therefore, these results potentially recommend the implementation of the HEC-PAA lyomatrix for intestinal targeting of GCV oral administration

\section{Analysis of the Physicomechanical Behavior of the Lyomatrix}

In order to estimate the GCV loading, the effect on the physicomechanical behavior of the lyomatrix textural analysis studies was conducted. MR attributed to the ability of the material to revert to its original structure after the application of a deforming force, and it is represented as a percentage of the ratio between the area under the compression and area under decompression curves (44). Textural analysis revealed that the MR, RG, and DE were dependent on the samples microstructure as shown in Fig. 5. The results indicated that the higher GCV concentration the lower DE. On the other hand, the higher drug concentrations the higher MR (\%) and RG. These results could be explained due to higher drug concentration causing a decrease in porosity as well as surface area (Table III), suggesting that there was a high correlation between the presence of GCV and the mechanical properties of the lyomatrix.

Table IV. Release Kinetics Parameters of Different GCV-Loaded Lyomatrix Formulations

\begin{tabular}{|c|c|c|c|c|c|c|c|c|c|c|}
\hline \multirow[b]{2}{*}{$\mathrm{pH}$} & \multirow[b]{2}{*}{ Formula code } & \multicolumn{2}{|c|}{ Zero-order } & \multirow{2}{*}{$\begin{array}{l}\text { Hopfenberg } \\
R^{2}\end{array}$} & \multicolumn{3}{|c|}{ Korsmeyer-Peppas } & \multicolumn{3}{|c|}{ Peppas-Sahlin } \\
\hline & & $t_{50}(\mathrm{~h})$ & $R^{2}$ & & $\mathrm{~K}$ & $n$ & $R^{2}$ & $\mathrm{~K}_{1}$ & $\mathrm{~K}_{2}$ & $R^{2}$ \\
\hline \multirow[t]{2}{*}{7.4} & LM 1 (10\% GCV) & 2.10 & 0.999 & 0.984 & 23.64 & 1.01 & 0.995 & 0.04 & 0.02 & 0.992 \\
\hline & LM 2 (20\% GCV) & 5.49 & 0.999 & 0.996 & 9.17 & 0.99 & 0.994 & 0.04 & 0.01 & 0.991 \\
\hline \multirow[t]{2}{*}{6.8} & LM 1 (10\% GCV) & 1.59 & 0.999 & 0.983 & 30.04 & 1.07 & 0.996 & 0.01 & 0.06 & 0.994 \\
\hline & LM 2 (20\% GCV) & 6.13 & 0.999 & 0.994 & 6.21 & 1.15 & 0.992 & 0.02 & 0.01 & 0.993 \\
\hline \multirow[t]{2}{*}{4.0} & LM 1 (10\% GCV) & 3.23 & 0.999 & 0.998 & 14.67 & 1.06 & 0.985 & 0.03 & 0.01 & 0.962 \\
\hline & LM $2(20 \%$ GCV $)$ & 11.74 & 0.999 & 0.997 & 4.34 & 1.01 & 0.993 & 0.01 & 0.01 & 0.973 \\
\hline \multirow[t]{2}{*}{1.2} & LM 1 (10\% GCV) & 9.25 & 0.999 & 0.999 & 5.02 & 1.04 & 0.997 & 0.01 & 0.01 & 0.991 \\
\hline & LM 2 (20\% GCV) & 58.18 & 0.999 & 0.999 & 1.07 & 0.87 & 0.996 & 0.01 & 0.01 & 0.995 \\
\hline
\end{tabular}

$t_{50}$ is the time required for $50 \%$ of the drug to be released and $R^{2}$ value is the value for regression coefficient 
Table V. Release Kinetics Parameters of Different GCV-Loaded Tablet Formulations

\begin{tabular}{|c|c|c|c|c|c|c|c|c|c|c|}
\hline \multirow[b]{2}{*}{$\mathrm{pH}$} & \multirow[b]{2}{*}{ Formula code } & \multirow{2}{*}{$\begin{array}{l}\text { Zero-order } \\
R^{2}\end{array}$} & \multirow{2}{*}{$\begin{array}{l}\text { Hopfenberg } \\
R^{2}\end{array}$} & \multicolumn{4}{|c|}{ Korsmeyer-Peppas } & \multicolumn{3}{|c|}{ Peppas-Sahlin } \\
\hline & & & & $\mathrm{K}$ & $n$ & $R^{2}$ & $t_{50}(\mathrm{~h})$ & $\mathrm{K}_{1}$ & $\mathrm{~K}_{2}$ & $R^{2}$ \\
\hline \multirow[t]{2}{*}{7.4} & Tablet $1(10 \% \mathrm{GCV})$ & 0.959 & 0.935 & 2.03 & 1.84 & 0.996 & 13.768 & 0.02 & 0.01 & 0.999 \\
\hline & Tablet $2(20 \% \mathrm{GCV})$ & 0.984 & 0.984 & 2.55 & 1.54 & 0.998 & 13.020 & 0.01 & 0.01 & 0.994 \\
\hline \multirow[t]{2}{*}{6.8} & Tablet $1(10 \%$ GCV $)$ & 0.983 & 0.968 & 4.63 & 1.39 & 0.998 & 8.000 & 0.01 & 0.01 & 0.998 \\
\hline & Tablet $2(20 \% \mathrm{GCV})$ & 0.992 & 0.984 & 3.72 & 1.5 & 0.999 & 9.307 & 0.01 & 0.02 & 0.992 \\
\hline \multirow[t]{2}{*}{4.0} & Tablet $1(10 \% \mathrm{GCV})$ & 0.966 & 0.978 & 3.26 & 1.62 & 0.993 & 9.827 & 0.03 & 0.01 & 0.998 \\
\hline & Tablet $2(20 \% \mathrm{GCV})$ & 0.970 & 0.983 & 1.74 & 1.73 & 0.997 & 17.016 & 0.02 & 0.01 & 0.999 \\
\hline \multirow[t]{2}{*}{1.2} & Tablet $1(10 \%$ GCV $)$ & 0.994 & 0.996 & 5.68 & 1.10 & 0.998 & 8.063 & 0.01 & 0.02 & 0.998 \\
\hline & Tablet $2(20 \% \mathrm{GCV})$ & 0.913 & 0.952 & 1.23 & 1.88 & 0.955 & 21.905 & 0.03 & 0.01 & 0.980 \\
\hline
\end{tabular}

Hydrational Swelling and Erosion of the GCV-Loaded Tablets via MRI

In order to investigate the relatively low GCV release from tablet formulation compared with the lyomatrix, MRI swelling and erosion monitoring studies were conducted on the GCV-loaded tablets and further qualitative confirmatory evidence. Figure 6 displays the MRI images obtained for the GCV-loaded tablets obtained at 0,4 , and $8 \mathrm{~h}$ corresponding to the GCV release time. The release medium (PBS: $\mathrm{pH} 1.2,4.0$, 6.8 , and 7.4) is represented by the light region surrounding the formulation. As the tablet swelled, the darker, un-hydrated portions of the formulation became white due to hydration as illustrated in Fig. 6c (29,45-47). The bioavailability of GCV after oral administration was studied by Anderson and coworkers (48). Their results confirmed that the degradation of GCV occurred under gastric $\mathrm{pH}$ conditions. Accordingly, the GCV-loaded tablets were assessed at both gastric and intestinal $\mathrm{pH}$ values in order to fully simulate the GIT milieu.

The erosion front and swelling regions were used to monitor the swelling rate of the lyomatrix tablets at different $\mathrm{pH}$ values. It is highlighted that the lowest gelling rate and rapid drug release was noticed at $\mathrm{pH} 6.8$ and 7.4 (Fig. 4f, h) due to the highest erosion rate. In contrast, the highest gelling rate and slowest drug release were observed at $\mathrm{pH} 1.2$ and 4.0 (Fig. 4b, d) due to the lowest erosion rate. These results were due to the $\mathrm{pH}$ sensitivity of the HEC-PAA lyomatrix based on the carboxylic group of PAA polymer $(42,43)$ as previously discussed under the drug release section. The made the erosion rate increase at neutral and slightly alkaline $\mathrm{pH}$ and decreased at acidic $\mathrm{pH}$ values. It is worthy to note that the tablets displayed a swelling and erosion resistance at acidic $\mathrm{pH}$ compared at neutral and slightly alkaline $\mathrm{pH}$ values. This result affirmed the success of the tablet in achieving intestinal targeting of GCV. However, the erosion rate was not sufficient to produce dissolution channels through the tablet that facilitated rapid zero-order GCV release as observed for the lyomatrix. These results could also be explained due to the highly porous nature of the lyomatrix, while the tablets possessed a more compact structure as confirmed by SEM. Thus porosity had a pertinent role in the hydration, swelling, and erosion of the lyomatrix to affect controlled drug release $(5,7,8)$.

\section{CONCLUSIONS}

A GCV-loaded lyomatrix was synthesized and investigated for its utility in intestinal targeting after oral administration. The lyomatrix provided rapid zero-order release kinetics of GCV compared to a tablet formulation. This was confirmed by Korsmeyer-Peppas release kinetic modeling. The success of intestinal targeting was dependent on $\mathrm{pH}$ sensitivity of the lyomatrix. This was proved by in vitro drug release and MRI hydration and erosion studies. The physicomechanical and micro-architectural properties were found to be dependent on the GCV concentration. These findings concluded that the lyomatrix is capable of delivering GCV to the intestine and the drug release behavior is regulated mainly by the microstructure of the lyomatrix. Although the lyomatrix produced by co-processing of two well-known biocompatible polymers is pharmaceutically characterized in this component of the work, detailed ex vivo and preclinical in vivo testing will be the next phase to confirm potential bioavailability enhancement of GCV.
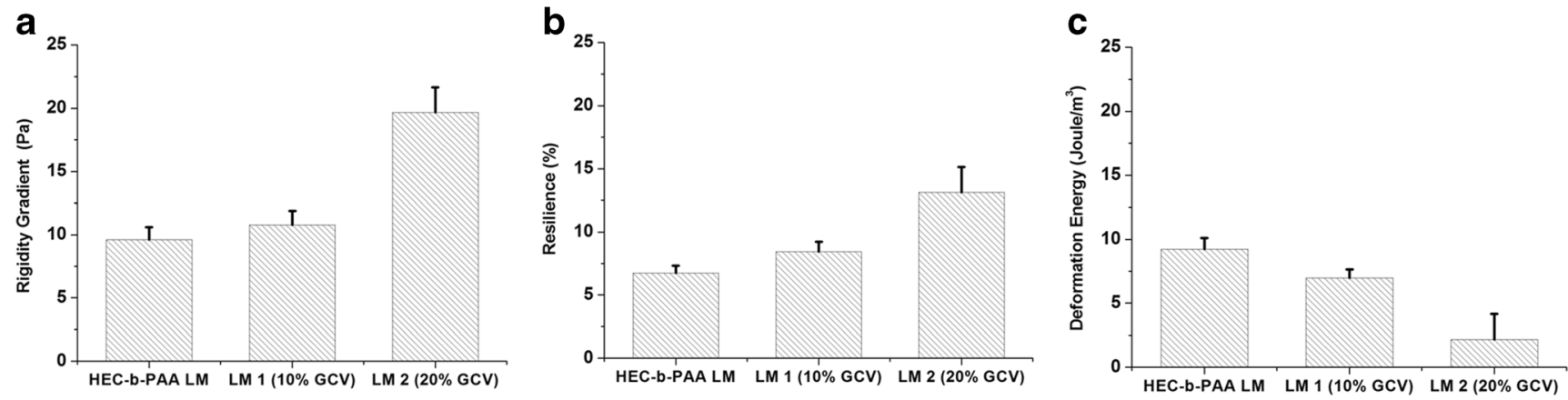

Fig. 5. Graphical representation of a rigidity gradient (RG), b matrix resilience (MR), and $\mathbf{c}$ deformation energy (DE) of HEC-PAA lyomatrix before and after GCV loading 


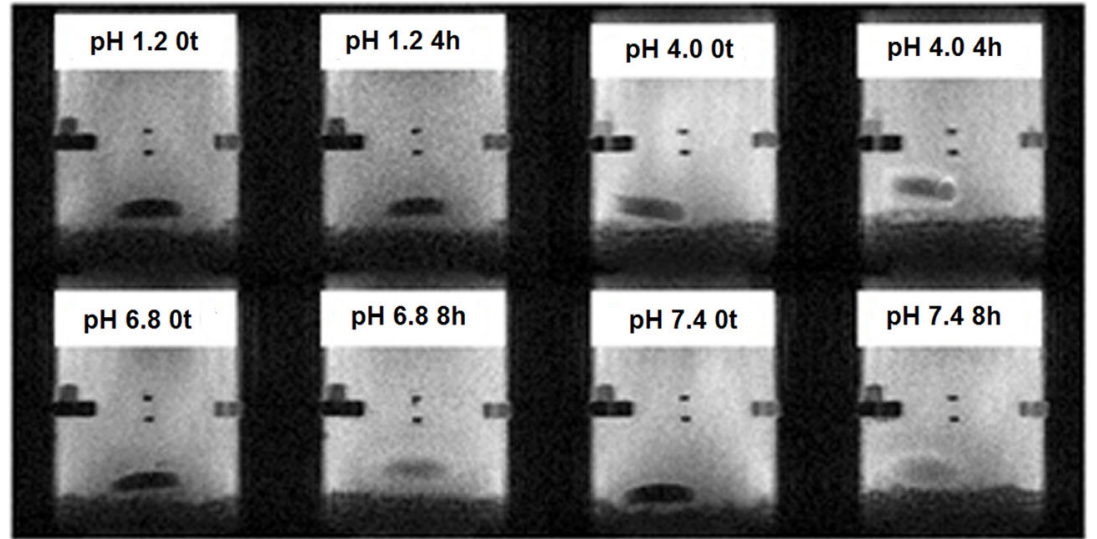

a

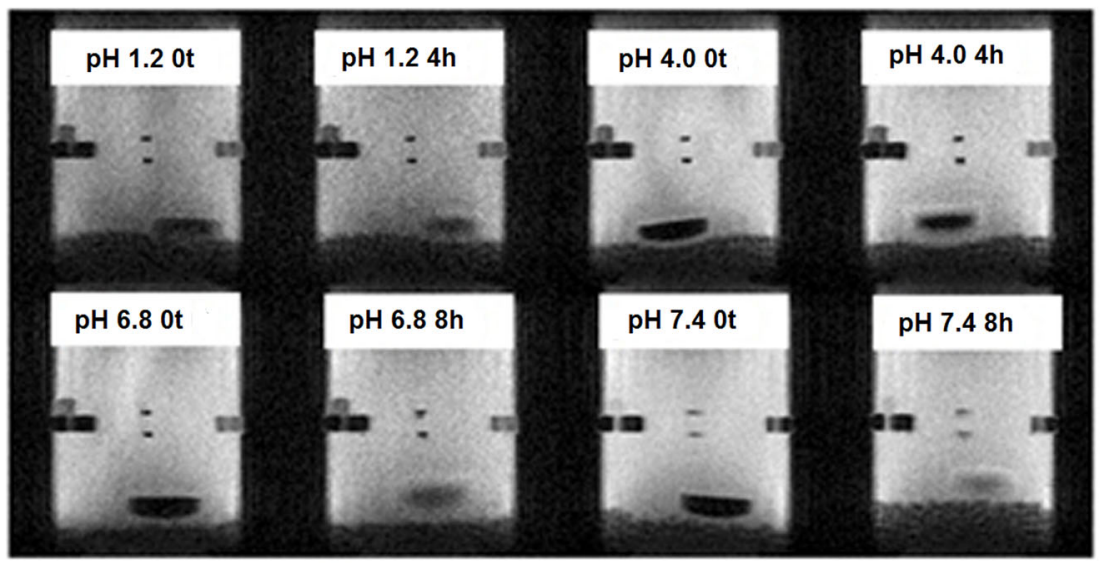

b

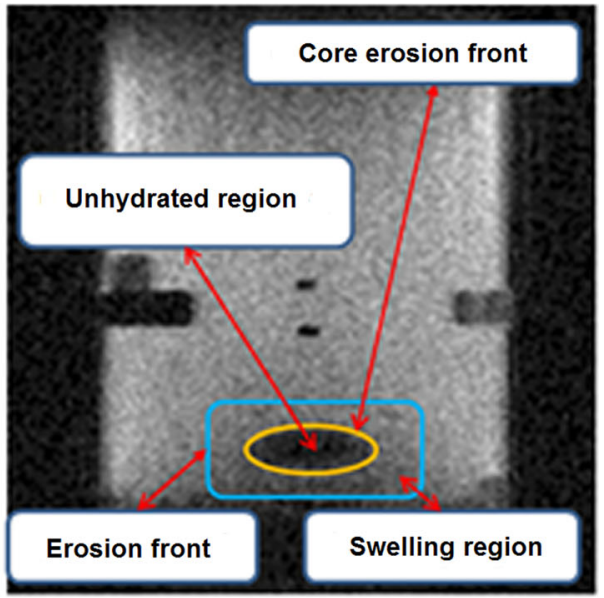

C

Fig. 6. MRI images of the hydration state of a tablet $1(10 \% \mathrm{GCV})$ and $\mathbf{b}$ tablet $2(20 \% \mathrm{GCV})$ at different $\mathrm{pH}$ values. $\mathbf{c}$ The position of the lyomatrix tablet during the MRI imaging denoting areas and fronts of swelling

\section{ACKNOWLEDGMENTS}

This work was funded by the National Research Foundation (NRF) of South Africa.

\section{COMPLIANCE WITH ETHICAL STANDARDS}

Conflict of Interest The authors declare that they have no competing interests.

\section{REFERENCES}

1. Gaby AR. Natural remedies for herpes simplex. Alternat Med Rev. 2006;11(2):93-101.

2. Avci O, Ertam I. Viral infections of the face. Clin Dermatol. 2014:32:715-33.

3. Alter SJ, Bennett JS, Koranyi K, Kreppel A, Simon R. Common childhood viral infections. Curr Probl Pediatr Ado Lesc Health Care. 2015;45:21-53.

4. Duvvuri S, Janoria KG, Mitra AK. Development of a novel formulation containing poly(d, l-lactide-co-glycolide) microspheres dispersed in PLGA-PEG-PLGA gel for sustained delivery of ganciclovir. J Control Release. 2005;108:282-93.

5. Gann MJ, Higginbotham CL, Geever LM, Nugent MJD. The synthesis of novel $\mathrm{pH}$-sensitive poly (vinyl alcohol) composite hydrogels using a freeze/thaw process for biomedical applications. Int J Pharm. 2009:372:154-61.

6. Kummara MR, Saravanan N, Deok JS, Chang-Sik H. pH sensitive halloysite-sodium hyaluronate/poly(hydroxyethyl methacrylate) nanocomposites for colon cancer drug delivery. Appl Clay Sci. 2014;97-98:33-42.

7. Wang Y, Chen L, Tan L, Zhao Q, Luo F, Wei Y, et al. PEG-PCL based micelle hydrogels as oral docetaxel delivery systems for breast cancer therapy. Biomaterials. 2014;35:6972-85.

8. Mukhopadhyay P, Sarkar K, Bhattacharya S, Bhattacharyya A, Mishra R, Kundu PP. pH sensitive N-succinyl chitosan grafted polyacrylamide hydrogel for oral insulin delivery. Carbohydr Polym. 2014;112:627-37.

9. Elliott JE, Macdonald M, Nie J, Bowman CN. Structure and swelling of poly(acrylic acid) hydrogels: effect of $\mathrm{pH}$, ionic strength, and dilution on the crosslinked polymer structure. Polymer. 2004;45:1503-10.

10. Craig DQM, Tamburic S, Buckton G, Newton JM. An investigation into the structure and properties of Carbopol®934 gels using dielectric spectroscopy and oscillatory rheometry. J Control Release. 1994;30:213-23.

11. Sannino A, Esposito A, Nicolais L, Del Nobile MA, Giovane A, Balestrieri $\mathrm{C}$, et al. Cellulose-based hydrogels as body water retainers. J Mater Sci-Mater Med. 2000;11(4):247-53.

12. Sannino A, Esposito A, De Rosa A, Cozzolino A, Ambrosio L, Nicolais L. Biomedical application of a superabsorbent hydrogel for body water elimination in the treatment of edemas. J Biomed Mater Res A. 2003;67(3):1016-24.

13. Esposito A, Sannino A, Cozzolino A, Quintiliano SN, Lamberti $\mathrm{M}$, Ambrosio L, et al. Response of intestinal cells and macrophages to an orally administered cellulose-PEG based polymer as 
a potential treatment for intractable edemas. Biomaterials. 2005;26(19):4101-10.

14. Zhang JT, Cheng SX, Huang SW, Zhuo R-X. Temperature sensitive poly(N-isopropylacrylamide) hydrogels with macroporous structure and fast response rate. Macromol Rapid Commun. 2003;24(7):447-51.

15. Zhang JT, Cheng SX, Zhuo RX. Preparation of macroporous poly ( $\mathrm{N}$-isopropylacrylamide) hydrogel with improved temperature sensitivity. J Polymer Scie Part A: Polymer Chem. 2003;41(15):2390-2.

16. Chen J, Park K. Synthesis and characterization of superporous hydrogel composites. J Control Release. 2000;65(1-2):73-82.

17. Hsieh DST, Rhine WD, Langer R. Zero-order controlled release polymer matrices for micro- and macromolecules. J Pharm Sci. 1983;72:17.

18. Korsmeyer RW, Peppas NA. In: Mansdorf SZ, Roseman TJ, editors. Controlled release systems. New York: Marcel Dekker; 1983. p. 77.

19. Zhang JT, Huang SW, Xue YN, Zhuo RX. Poly (Nisopropylacrylamide) nanoparticle-incorporated PNIPAAM hydrogels with fast shrinking kinetics. Macromol Rapid Commun. 2005;26(16):1346-50.

20. Zhang XZ, Zhuo RX. A novel method to prepare a fast responsive, thermosensitive poly ( $\mathrm{N}$-isopropylacrylamide) hydrogel. Macromol Rapid Commun. 1999;20(4):229-31.

21. Kato N, Sakai Y, Shibata S. Wide-range control of deswelling time for thermosensitive poly (N-isopropylacrylamide) gel treated by freeze-drying. Macromolecules. 2003;36(4):961-3.

22. Sophie R, Mies J, Stefaan C. Self-gelling hydrogels based on oppositely charged dextran microspheres. Biomaterials. 2005;26:2129-235.

23. Pillay V, Fassihi R. A novel approach for constant rate delivery of highly soluble bioactives from a simple monolithic system. J Control Release. 2000;67:67-78.

24. Choonara YE, Pillay V, Carmichael T, Danckwerts MP. An in vitro study of the design and development of a novel doughnut-shaped minitablet for intraocular implantation. Int J Pharm. 2006;3:15-24.

25. Ritger PL, Peppas NA. A simple equation for description of solute release. II swellable devices. J Control Release. 1987;5:37-42.

26. Kim H, Fassihi R. Application of binary polymer system in variables and hydrodynamic conditions on release kinetics. J Pharm Sci. 1997;86:323-8.

27. Peppas NA, Sahlin JJ. A simple equation for the description of solute release. III. Coupling of diffusion and relaxation. Int J Pharmceutics. 1989;57:169-72.

28. Zhang Y, Huo M, Zhou J, Xie SPK. Solver: an add-in program for pharmacokinetic and pharmacodynamic data analysis in Microsoft Excel. Comput Methods Prog Biomed. 2010;99(3):306-14.

29. Indermun S, Choonara YE, Kumar P, du Toit LC, Modi G, Luttge $\mathrm{R}$, et al. An interfacially plasticized electro-responsive hydrogel for transdermal electro-activated and modulated (TEAM) drug delivery. Int J Pharm. 2014;462:52-65.

30. Dhirendra K, Lewis S, Udupa N, Atin K. Solid dispersion: a review, Pak. J Pharm Sci. 2009;22(2):234-46.

31. AL-Kahtania AA, Sherigara BS. Controlled release of diclofenac sodium through acrylamide grafted hydroxyethyl cellulose and sodium alginate. Carbohydr Polym. 2014;104:151-7.

32. Yu-Li L, Yijun L, Hong-Ru L. Evaluation of epirubicin in thermogelling and bioadhesive liquid and solid suppository formulations for rectal administration. Int J Mol Sci. 2014;15(1):342-60.

33. Mabrouk M, Mostafa AA, Oudadesse H, Mahmoud AA, ElGohary MI. Effect of ciprofloxacin incorporation in PVA and PVA bioactive glass composite scaffolds. Ceram Int. 2014;40:4833-45.

34. Ghosh T, Auerochs S, Saha S, Ray B, Marscha M. Anticytomegalovirus activity of sulfatedglucans generated from a commercial preparation of rice bran. Antivir Chem Chemother. 2010;21:85-95.

35. Chen X, Ooi CP, Lim TH. Effect of ganciclovir on the hydrolytic degradation of poly(lactide-co-glycolide) microspheres. J Biomater Appl. 2006;20:287-302.

36. Hosseinzadeh H. Controlled release of diclofenac sodium from pH-responsive carrageenan-g-poly(acrylic acid) superabsorbent hydrogel. J Chem Sci. 2010;122(4):651-9.

37. Wiedemair J, Serpe MJ, Kim J, Masson JF, Lyon LA, Mizaikoff $\mathrm{B}$, et al. In situ AFM studies of the phase-transition behavior of single thermoresponsive hydrogel particles. Langmuir. 2007;23:130-7.

38. Mizuguchi Y, Koga T, Higashi N. Supramolecular nanoarchitectures from fullerene-agged sequential peptides by self-assembly, polymers. Prepr Jpn. 2006;55:1890.

39. Sahiner N, Godbey WT, Pherson GLM, John VT. Microgel, nanogel and hydrogel semi-IPN composites for biomedical applications: synthesis and characterization. Colloid Polym Sci. 2006;284:1121-9.

40. Matsukuma D, Yamamoto K, Aoyagi T. Stimuli-responsive properties of $\mathrm{N}$-isopropyl acrylamide-based ultrathin hydrogel films prepared by photo-cross-linking. Langmuir. 2006;22:5911-5.

41. Ganesh M, Lee SG. Synthesis, characterization and drug release capability of new cost effective mesoporous silica nano particle for ibuprofen drug delivery. Int j Control Autom. 2013;6(5):20716.

42. Milosavljevi'c BN, Ristic ME, Peri'c-Gruji AA, Filipović JM, Strbac SB, Rakočević ZL, et al. Hydrogel based on chitosan, itaconic acid and methacrylic acid as adsorbent of $\mathrm{Cd}^{2+}$ ions from aqueous solution. Chem Eng J. 2010;165:554-62.

43. Po R. Water-absorbent polymers: a patent survey. J Macromol Sci Rev Macromol Chem Phys. 1994;C34:607-61.

44. Kost J. In: Mathiowitz E, editor. Encyclopedia of controlled drug delivery. 1st ed. New York: Wiley; 1999. p. 445.

45. Mikac U, Sepe A, Krist J, Baumgartner S. A new approach combining different MRI methods to provide detailed view on swelling dynamics of xanthan tablets influencing drug release at different $\mathrm{pH}$ and ionic strength. J Control Release. 2010;145:24756.

46. Chung E, Stenmark MH, Evans C, Narayana V, McLaughlin PW. Greater postimplant swelling in small volume prostate glands: implications for dosimetry, treatment planning, and operating room technique, Int. J Radiat Oncol Biol Phys. 2012;82(5):19448.

47. Dvinskikh SV, Szutkowski K, Furó I. MRI profiles over very wide concentration ranges: application to swelling of a bentonite clay. $\mathrm{J}$ Magn Reson. 2009;198:146-50.

48. Anderson RD, Grim KG, Jung D, Dorq A, Hulse J, Smith RB. Ganciclovir absolute bioavailability and steady-state pharmacokinetics after oral administration of two $3000-\mathrm{mg} / \mathrm{d}$ dosing regimens in human immunodeficiency virus- and cytomegalovirusseropositive patients. Clin Ther SW. 1995;17(3):425-32. 